\title{
A Science Based Approach to Topical Drug Classification System (TCS)
} \author{
Vinod P. Shah ${ }^{1,5}$, Avraham Yacobi ${ }^{2}$, Flavian Ștefan Rădulescu ${ }^{3}$, Dalia Simona Miron ${ }^{3}$, Majella E. \\ Lane $^{4}$
}

ABSTRACT

The Biopharmaceutics Classification System (BCS) for oral immediate release solid drug products have been very successful; its implementation in drug industry and regulatory approval has shown significant progress. This has been the case primarily because BCS was developed using sound scientific judgment. Following the success of BCS, we have considered the topical drug products for similar classification system based on sound scientific principles.

In USA, most of the generic topical drug products have qualitatively (Q1) and quantitatively (Q2) same excipients as the reference listed drug (RLD). The applications of in vitro release (IVR) and in vitro characterization are considered for a range of dosage forms (suspensions, creams, ointments and gels) of differing strengths. We advance a Topical Drug Classification System (TCS) based on a consideration of Q1, Q2 as well as the arrangement of matter and structure of topical formulations (Q3). Four distinct classes are presented for the various scenarios that may arise and depending on whether biowaiver can be granted or not.

KEY WORDS: Bioequivalence, Semi-solids, Skin, In vitro models, Regulatory sciences.

\section{INTRODUCTION}

\footnotetext{
${ }^{1}$ Pharmaceutical Consultant, North Potomac, Maryland, USA.

${ }^{2}$ DOLE Pharma LLC, Englewood, New Jersey, USA.

${ }^{3}$ Faculty of Pharmacy, University of Medicine and Pharmacy "Carol Davila”, Bucharest, Romania.

${ }^{4}$ University College London School of Pharmacy, London, UK.

${ }^{5}$ To whom correspondence should be addressed. (e-mail: dr.vpshah@comcast.net)
} 
Topical dermatological products are liquid or semisolid preparations such as creams, ointments, lotions and gels. They deliver drugs to various layers of the skin to prevent or treat diseases. The onset, duration and magnitude of therapeutic response for any topical formulation depends on the relative efficiency of three sequential processes: release of the active drug substance from the dosage form (drug release), penetration/diffusion of the drug through the stratum corneum and other layers of skin before eliciting the desired pharmacological effect at the site of action. These processes are variable which results in differences in the safety and efficacy of the formulation. Ultimately such variability results in serious challenges in the determination of bioequivalence (BE) of topical dermatologic products, challenges which encumber the development of acceptable generic formulations by the pharmaceutical industry and their approval by regulatory authorities.

In the US a generic product is required to be both pharmaceutically equivalent (PE) and BE. PE means that the generic product has the same active ingredient in the same amount and same type of dosage form. In addition, according to 21 CFR 314.94 for topical dosage forms (2013), the generic product will need to have the same excipients and be qualitatively (Q1) and quantitatively (Q2) the same as the RLD. When a generic product is approved it is considered therapeutically equivalent and interchangeable with the brand name product. BE of a topical dosage form is documented by comparing the test and reference products using pharmacokinetic, pharmacodynamic, clinical or in vitro tests. Currently, comparative clinical endpoint trials are used to establish BE for most dermatological drug products except for glucocorticoids which are tested via an established pharmacodynamic test (US-FDA, 1997). Clinical trials require the demonstration of BE of the generic drug to the RLD, using one or more clinical endpoints and assuring efficacy by establishing superiority of the generic formulation and RLD over placebo. These trials with vigorous statistical requirements are associated with a high degree of variability and low sensitivity to formulation factors which means that they are comparatively less efficient and in some cases not conclusive. In order to meet a minimum statistical power to show efficacy and BE of a generic product and RLD reliably, a clinical trial with enrollment of a large number of patients will be needed. Such trials are often tedious, expensive and sometimes impossible to complete. Because of these reasons, it can be 
concluded that in many cases the regulatory requirements for generic topical drug products are complex, cumbersome, and time consuming.

The FDA has recently issued two draft guidances for generic acyclovir ointment (US-FDA, 2012) and cyclosporine ophthalmic emulsion (US-FDA, 2013) including an in vitro option to establish BE, provided a set of qualification criteria are met. This development is viewed with optimism by the authors to offer the opportunity for new initiatives, relying on comparative physico-chemical evaluation of topical semisolids and in vitro release (IVR) testing.

During a recent BE workshop on Current challenges in bioequivalence, quality, and novel assessment technologies for topical products (Yacobi et al., 2014), opportunities to simplify the regulatory requirements have been identified. Considering the science developed for Scale Up and Post Approval Changes for nonsterile Semisolid Dosage Forms (SUPAC-SS, 1997) and the principles of IVR, a biowaiver based on rigorous in vitro characterization of the dosage form compared with the RLD is feasible (US-FDA, 2012; 2013). The draft guidelines of acyclovir ointment and cysclosporin ophthalmic emulsion are an extension of SUPAC-SS guidance based on well-established and time honored scientific principles. More importantly, there is no evidence suggesting that the application of approaches identified in SUPAC-SS compromise the quality of topical dosage forms. The IVR is recognized as the most important test to assure the quality of the drug product. Herein we enunciate practical applications of science based principles of SUPAC-SS combined with the use of IVR methods for regulatory evaluation of topical drug products and propose the concept of developing a relevant "Topical Drug Classification System (TCS)" which may be used by both the pharmaceutical industry and

75 regulatory agencies across the world.

\section{IN VITRO DRUG RELEASE}

The efficacy of a topically applied dosage form may be dependent on drug release, therefore the IVR rate (profile) theoretically constitutes an important and valuable product quality parameter. The development of simple, reliable, reproducible and validated methods to determine the drug release from a semisolid dosage form using a vertical diffusion cell (VDC) system and a synthetic membrane was reported (Shah et al., 2003; Hauck et al., 2007). The 
authors' preference is to use VDC for IVR determination, because of long experience and adequate standardization with this apparatus. However, other methods are also described (USP37-NF32, 2014).

\section{SCALE UP AND POST APPROVAL CHANGES (SUPAC-SS)}

The topical semisolid drug products are complex formulations with complex structural configurations. The API is either dissolved or dispersed in the vehicle. The physical properties of the dosage form are dependent on various factors, including the size of the dispersed particles, arrangement and interactions between the dispersed materials, the particle size of the API, the interfacial tension between the phases, the partition coefficient of the drug between the phases, and the product rheology.

The SUPAC-SS guidance (1997) was developed to address (i) changes in the component or composition (ii) changes in the manufacturing process and equipment (iii) the scale-up/scaledown of manufacture, and/or change in site of manufacture of a semisolid formulation during the post-approval period. The Level 2 change can include: (i) changes of $>5$ and $\leq 10 \%$ of excipients (ii) change in equipment to a different design or different operating principles; process changes including changes in rate of mixing, rate of cooling, operating speeds and holding time (iii) change in batch size beyond a factor of 10 .

The IVR is recommended to assure that consistent product performance will be achieved after Level 2 changes. The IVR of the changed product must be the same as for the initially approved drug product. In this scenario, IVR is used as a measure of equivalence in product performance, and is used to requalify the initially approved product after an acceptable change. Thus, the IVR is considered to be a useful test to assess product sameness between pre-change and post-change product. Moreover, IVR test has also been recognized as a reasonable and useful test to consider for product release and stability testing (Chang et al., 2013). In vitro nonsimilarity could be a signal of altered in vivo performance (SUPAC-SS, 1997; USP37-NF32, 2014).

Direct confirmation of structural equivalency is difficult to obtain where a formulation is 110 not a solution (Lionberger, 2004). Available reports suggest that Q1 and Q2 equivalent products, also bioequivalent as demonstrated by clinical endpoint studies, displayed Q3 
difference (spreadability differences; Kryscio et al., 2008). Therefore, structural analysis alone cannot provide adequate information on the first step of the release process, the diffusion through the semisolid matrix. We consider feasible to rely on the use of simple, reliable and reproducible IVR methodology for the evaluation of Q3 similarity, i.e. the same state of aggregation and the same arrangement of the matter or microstructure. When adequately developed and validated, IVR methodology can provide information on the combined role of several physico-chemical characteristics, including the particle or droplet size, viscosity and diffusional resistance of the vehicle.

After extensive research, Klein and coworkers (2010) confirmed the principles of SUPACSS guidance (1997) and statements of the 1999 AAPS-FDA workshop report (Flynn et al., 1999). Pillai et al (2001) also confirmed that the IVR methodology is a feasible, reliable "product qualifying tool", the release rates being sensitive indicators of the "state of solubilization of a drug, to the drug's particle size, to the method and rigor of drug distribution, and to other factors of system composition and processing" (Flynn et al., 1999).

\section{WAIVER FOR LOWER STRENGTHS OF TOPICAL DOSAGE FORMS}

In the case of multi strength dosage forms of tablets and capsules, an in vivo BE study comparing the highest potency of test and RLD product is required. For the lower strengths a biowaiver is allowed, provided that the additional strengths are formulation proportional or pseudo-proportional for high potency drug, have the same drug releasing mechanism and have similar drug release profiles when compared to the highest strength of the bioequivalent product (US-FDA, 2003). Generally, different strengths of topical dosage forms from a given manufacturer differ only in the amount of active ingredient, the basic formulation or vehicle is

135 the same for all strengths. This general similarity between oral and topical dosage forms is shown in Figure 1.

Figure 1. 
As an example, assume that two strengths differ by $50 \%$, i.e., they are $0.5 \%$ and $0.25 \%$. The IVR for the lower strength will depend on the status of the active ingredient, either dissolved or dispersed; if it is in solution form, the IVR for the lower strength will be half that of the higher strength, and if it is in suspension form, the IVR for the lower strength will be proportional to the square root of 2 (Pillai et al., 2001). The IVR ratio for the two strengths will be equal to the ratio of the amount or concentration of the dissolved active pharmaceutical ingredient. In the case of the dispersed system, the IVR will be equal to the square root of the amount or concentration of the dissolved active pharmaceutical ingredient. These relationships provide a framework for allowing a biowaiver for lower strengths of the topical dosage form produced by the same manufacturer.

\section{BIOPHARMACEUTICS CLASSIFICATION SYSTEM (BCS)}

The Biopharmaceutics Classification System (BCS) for immediate release solid oral dosage form is based on the properties of the API, its aqueous solubility and intestinal permeability (US-FDA, 2000). This scientifically sound framework has gained wide regulatory acceptance and allows the use of in vitro dissolution testing as alternative for in vivo BE studies, provided a set of clearly defined solubility, permeability and dissolution criteria are met. Generic immediate release dosage forms of the BCS class 1 and class 3 drugs are eligible for biowaiver, if dissolution criteria are met when compared to the RLD. The validity of the principles underlying the BCS classification has been confirmed based on its utilization in drug development in industry and its implementation in regulatory approval process. BCS class 2 and 4 are not eligible for biowaiver and require in vivo BE studies.

\section{TOPICAL DRUG CLASSIFICATION SYSTEM (TCS)}

The proposed topical drug classification system is based on qualitative and quantitative equivalence of composition (Q1 and Q2) and on the similarity of IVR rates (as estimator of microstructural sameness, Q3) between two compared formulations, a generic product and RLD. The excipients in topical dosage forms can play an active role in the in vivo performance of 
the formulations compared to immediate release oral dosage forms. Depending on the nature and quantity, the excipients may have a significant impact on the release from the dosage form, on the skin barrier properties and drug penetration, directly affecting rate and extent of exposure at site of action. The IVR (Q3) reflects the microstructure, arrangement of the matter and the state of aggregation of the dosage form. If all three parameters, Q1, Q2 and Q3 are the same between the RLD and the generic product, the generic product may be suitable for a 175 biowaiver. If they are not the same, then of course, biowaiver cannot be provided and additional studies or a biostudy will be required. Using these scientific principles, a topical drug classification system (TCS) is proposed to simplify the regulatory requirements for topical dosage forms, and is shown in Figure 2.

Based on composition and IVR similarity, the compared dosage forms are classified as TCS class 1, 2, 3 and 4. Under the proposed classification, only TCS class 1 and TCS class 3 dosage forms are eligible for biowaiver; TCS class 2 and TCS class 4, are not eligible for biowaiver and will require in vivo BE studies for drug approval. The nature and type of in vivo BE study will depend on the therapeutic class and dosage form category. The proposed TCS is as follows:

- If the product is Q1 and Q2, and if it meets IVR (Q3) comparison criteria and confidence intervals identified in SUPAC-SS, a biowaiver can be provided. This may be classified as TCS class 1 and corresponds to the definition of Level 1 changes in the SUPAC-SS guidance. There is no reason to expect the generic product to perform differently than the RLD under such a scenario.

- If the product is Q1 and Q2, but has different Q3, then a biowaiver cannot be granted, and an appropriate BE study should be required. This may be classified as TCS class 2 .

- If the generic product is not Q1 and Q2, then it necessitates evaluation of the excipients, to determine if they are inert or not inert. Excipients can influence drug penetration and may have an effect on in vivo performance of the product, thereby changing the safety and efficacy profiles. It is therefore essential to evaluate the properties of the excipients 
with respect to safety and efficacy, as well as how excipients affect both the thermodynamic activity of the active pharmaceutical ingredient and the skin permeability. In addition, the IVR needs to be determined. If the IVR turns out to be the same as the RLD, and meets the confidence interval criteria, then the dosage form can be provided with a biowaiver. This may be classified as TCS class 3 .

- If the generic product is not Q1 and Q2, and IVR is different, then biowaiver cannot be granted, and an appropriate in vivo study will be required for topical drug product approval. This may be classified as TCS class 4.

\section{DISCUSSION}

The BCS for solid oral dosage forms is based on sound scientific principles and has reduced regulatory burden and avoided unnecessary in vivo study requirements for a number of drug products. The BCS has been in place for over a decade and has resulted in considerable success in facilitating the development and approval of a larger number of generic products with considerable savings to the consumer. These API products with high solubility and permeability characteristics are approved based on dissolution studies.

Regulatory requirements for generic topical drug products are complex and cumbersome,

215 time consuming and at times questionable as they prevent the possibility of developing alternative and more affordable products. Recently, the FDA has allowed the utilization of accepted and validated in vitro methods for generic approval of at least two products: acyclovir ointment and cyclosporine ophthalmic emulsion. Conducting reliable and successful clinical end-point bioequivalence studies for these products was near to impossible. However, the consideration of in vitro tests for topical products in lieu of clinical studies offered the opportunity to explore the application of such tests to other products which have been considered "untouchables" for development by generic firms. Therefore, the development of a science based approach to elucidate the requirements for the development of safe, effective and interchangeable topical generic drug products should play a key role to advance the 225 approval of generic products which have enjoyed unintended protection from competition. The purpose of this communication is to offer possible use of an approach similar to the BCS that 
may be referred to as the TCS system which is also based on established scientific principles specifically developed for semisolid topical products. Unlike the officially adopted BCS which is based on the solubility and permeability characteristics of the drug, the proposed TCS considers the qualitative and quantitative composition of inactive ingredients and microstructure arrangement of topical semisolid products. In both the classification systems, their applicability for biowaiver granting relies on the use of in vitro methodologies as key decision tools.

The principles are in-line with the previous proposal of a decision tree, for drugs targeting 235 the stratum corneum (Lionberger, 2004). If products were Q1 and Q2 equivalent, IVR testing was recommended, with in vivo testing waived based on the conclusion of similarity, this corresponds to the present approach for TCS class 1. For products Q1 equivalent with Q2 differences, IVR was suggested, supplemented by in vivo evaluations (defined as potential alteration of either skin permeability or solubility of drug in the formulation). In case of Q1 and Q2 non-equivalencies, IVR and in vivo approaches were to be required in order to demonstrate the lack of a formulation effect on absorption.

The SUPAC-SS guidance which provides assurance for product sameness between approved and post-approval product, has been in place for almost two decades and is based on IVR principles. This criteria was expanded further with Q1 and Q2 criteria, with added Q3 requirements, indicating that with $\mathrm{Q} 1$ and $\mathrm{Q} 2$ being the same, a generic product so manufactured falls into the category of SUPAC-SS Level 2 changes. If IVR (Q3) is the same, indicating a strong likelihood that the formulation process confers similar product structure, a biowaiver may be granted. Using the well-established BCS schematic, the TCS schematic is presented in Figure 3. If a similar system is considered, it will allow the use of already established and utilized methodologies which will significantly reduce the burden for generic topical drug product development and approval, without sacrificing the product quality or compromising the safety and efficacy of potential generic products.

\section{Figure 3.}


All the changes under SUPAC-SS refer to the initially approved composition or process and the possible impact on the in vivo performance. The level 2 changes are evaluated by adequately developed and validated IVR methodology. The topical semisolid products included in the TCS classes 1 and 3 also can be assimilated to one of these two levels of changes under

260 SUPAC-SS. There is no scientific reason to believe that the assumed risks of the waiving procedures are higher in the approval of a generic topical semisolid product under the TCS principles, compared to the post-approval changed formulation under SUPAC-SS. SUPAC-SS guidance allows for a maximum $10 \%$ change in the quantity of any or collectively, all excipients, by applying IVR tests. The same principles are suggested to be implemented for TCS class 3 drug

265 products. Any quantitative changes outside the previously mentioned ranges are to be analyzed for their in vivo significance. Only in cases where the lack of significant impact of safety and efficacy profiles is concluded, the IVR (Q3) should be used as the basis of waiving the in vivo demonstration of bioequivalence. IVR (Q3) differences will indicate that alternative methods must be considered, as pointed out in the recently reported "Decision Tree Strawman for Determination of Topical Bioequivalence" (Yacobi et al., 2014). It is to be noted that the currently described TCS framework, based on Q1, Q2 and Q3 similarities, represents a particularization of the first tier of the decision tree. This classification system is based on the composition and characteristics of the topical semisolid dosage forms and uses the IVR methodology as a product qualifying tool, similar to SUPAC-SS. The difference resides on the

275 extended applicability of IVR, beyond the current role of supportive, not surrogate methodology in the assessment of bioequivalence.

\section{CONCLUSION}

A practical and science based classification system, TCS, for topical drug products is proposed that will facilitate generic product development, reduce the regulatory burden and assure product quality. This will facilitate the development of topical generic products across all therapeutic classes, at all strengths at which the RLD is marketed, their approval, and ultimately their availability to patients and consumers at a more reasonable cost. 


\section{ACKNOWLEDGEMENT}

The authors would like to thank Dr. Jonathan Hadgraft, University College London, School of Pharmacy, London, UK for his critical comments on the manuscript. 


\section{REFERENCES}

21 CFR 314.94, 2013. Code of Federal Regulations. Title 21 - Food and drugs. Chapter I - Food and drug administration department of health and human services subchapter D - Drugs for human use. Part 314 - Applications for FDA approval to market a new drug. Subpart C -

Abbreviated Applications. Sec.314.94 Content and format of an abbreviated application. 2013. Accessed April 18, 2015, at:

295 http://www.accessdata.fda.gov/scripts/cdrh/cfdocs/cfcfr/cfrsearch.cfm?fr=314.94.

Chang, R.K., Raw, A., Lionberger, R., Yu, L., 2013. Generic development of topical dermatologic products: formulation development, process development, and testing of topical dermatologic products. AAPS J. 15, 41-52.

Flynn, G.L., Shah, V.P., Tenjarla, S.N., Corbo, M., DeMagistris, D., Feldman, T.G., Franz, T.J.,

Miran, D.R., Pearce, D.M., Sequeira, J.A., Swarbrick, J., Wang, J.C., Yacobi, A., Zatz, J.L., 1999.

Assessment of value and applications of in vitro testing of topical dermatological drug products. Pharm. Res. 16, 1325-1330.

Hauck, W.W., Shah, V.P., Shaw, S.W., Ueda, C.T., 2007. Reliability and reproducibility of vertical diffusion cells for determining release rates from semisolid dosage forms. Pharm. Res. 24, 20182024.

Klein, R.R., Tao, J.Q., Wilder, S., Burchett, K., Bui, Q., Thakker, K.D., 2010. Development of an In Vitro Release Test (IVRT) for a Vaginal Microbicide Gel. Diss. Tech. 17, 6-10.

Kryscio, D.R., Sathe, P.M., Lionberger, R., Yu, L., Bell, M.A., Jay, M., Hilt, J.Z., 2008. Spreadability measurements to assess structural equivalence (Q3) of topical formulations- technical note.

310 AAPS PharmSciTech. 9, 84-86.

Lionberger, R., 2004. Presentation at the Advisory Committee meeting for Pharmaceutical Sciences, April 14, 2004. Accessed April 18, 2015, at:

http://www.fda.gov/ohrms/dockets/AC/04/slides/4034S2_12_Lionberger.ppt.

Pillai, R., Shah, V., Abriola, L., Caetano, P., Flynn, G.L., 2001. Release of hydrocortisone from a cream matrix: dependency of release on suspension concentration and measurement of solubility and diffusivity. Pharm. Dev. Technol. 6, 373-384. 
Shah, V.P., Elkins, J., Shaw, S., Hanson, R., 2003. In vitro release: comparative evaluation of vertical diffusion cell system and automated procedure. Pharm. Dev. Technol. 8, 97-102.

SUPAC-SS, 1997. US Department of Health and Human Services, Food and Drug Administration. 1997. Guidance for Industry. SUPAC-SS. Nonsterile semisolid dosage forms. Scale-up and post approval changes. Chemistry, manufacturing and controls. In vitro release testing and in vivo bioequivalence documentation. Accessed April 18, 2015, at: http://www.fda.gov/cder/guidance/index.htm. US-FDA, 1997. US Department of Health and Human Services, Food and Drug Administration.

325 1997. Guidance for industry: Topical Dermatological Corticosteroids. In Vivo Bioequivalence. Accessed April 18, 2015, at:

http://www.fda.gov/downloads/Drugs/GuidanceComplianceRegulatorylnformation/Guidances/ ucm070234.pdf.

US-FDA, 2000. US Department of Health and Human Services, Food and Drug Administration.

330 Guidance for Industry. Waiver of in vivo bioavailability and bioequivalence studies for immediate-release solid oral dosage forms based on a Biopharmaceutics Classification System. 2000. Accessed April 18, 2015, at:

http://www.fda.gov/downloads/Drugs/Guidances/ucm070246.pdf US-FDA, 2003. US Department of Health and Human Services, Food and Drug Administration. 2003. Guidance for Industry. Bioavailability and Bioequivalence Studies for Orally Administered Drug Products - General Considerations. March 2003 BP Revision 1. Accessed April 18, 2015, at: http://www.fda.gov/downloads/Drugs/Guidances/ucm070124.pdf. US-FDA, 2012. Food and Drug Administration, Office of Generic Drugs. 2012. Draft guidance on acyclovir ointment. Accessed April 18, 2015, at:

340 http://www.fda.gov/downloads/Drugs/GuidanceComplianceRegulatorylnformation/Guidances/ ucm296733.pdf.

US-FDA, 2013. Food and Drug Administration, Office of Generic Drugs. 2013. Draft guidance on cyclosporine ophthalmic emulsion. Accessed April 18, 2015, at:

http://www.fda.gov/downloads/Drugs/GuidanceComplianceRegulatoryInformation/Guidances/ ucm358114.pdf. 
USP37-NF32, 2014. United States Pharmacopoeia 37 - National Formulary 32, 2014. Chapter

1724: Semisolid Drug Products - Performance Tests, United States Pharmacopeial Convention, Rockville, MD 20852. 1273-1284.

Yacobi, A., Shah, V.P., Bashaw, E.D., Benfeldt, E., Davit, B., Ganes, D., Ghosh, T., Kanfer, I., 350 Kasting, G.B., Katz, L., Lionberger, R., Lu, G.W., Maibach, H.I., Pershing, L.K., Rackley, R.J., Raw, A., Shukla, C.G., Thakker, K., Wagner, N., Zovko, E., Lane, M.E., 2014. Current challenges in bioequivalence, quality, and novel assessment technologies for topical products. Pharm. Res. 31, 837-846. 
Figure 1: Schematics of Biowaiver for Oral Immediate Release and Topical Semisolid Dosage Forms

Figure 2: Proposed Decision Tree in Granting Biowaivers for Topical Dosage Forms

Figure 3: Topical Drug Classification System, TCS 